\title{
EL CONGRESO NACIONAL Y LOS PROYECTOS PARA LA CONSTRUCCIÓN DE LOS FERROCARRILES EN EL LITORAL. CORRIENTES Y ENTRE RÍOS, 1862-1880**
}

\author{
National Congress and projects for railways construction in the Litoral. \\ Corrientes and Entre Ríos, 1862-1880
}

Raquel V. Bressan*

\section{Resumen}

El presente artículo aborda el desarrollo de los proyectos ferroviarios para las provincias de Corrientes y Entre Ríos durante la etapa de construcción del Estado-Nación. El análisis se centra en los debates parlamentarios que tuvieron lugar durante las décadas de 1860 y 1870 e indaga cuál fue el rol desempeñado por los legisladores y por diversos actores, a nivel nacional y provincial, en la promoción de los ferrocarriles diseñados para estas provincias. A partir del examen de fuentes como los diarios de sesiones de la cámara de diputados y senadores, correspondencia particular y oficial de los funcionarios públicos y artículos periodísticos, se busca repensar los vínculos establecidos entre el Estado nacional y las elites provinciales -planteados por estudios ya clásicos como relaciones de cooptación- y mostrar las interacciones y conflictos entre las instituciones nacionales y provinciales que arbitraron la dirección de las políticas públicas en las provincias del litoral.

$$
<\text { Congreso }><\text { Ferrocarriles }><\text { Estado-Nación }><\text { Provincias }>
$$

\footnotetext{
Abstract

This article discusses the development of railway projects in the provinces of Corrientes and Entre Rios during the building of the Nation State. The analysis focuses on the parliamentary debates that took place during the decade of 1860 and 1870, and examines the role played by legislators and different actors, from the national and provincial levels, in promoting the railways designed for these provinces. Based on the analysis of sources such as the sessions of the chamber of deputies and senators, official and private correspondence and newspaper articles, it seeks to rethink the links established between the national government and the provincial elites -regarded by previous studies as cooptation relationships. In addition, it shows the interactions and conflicts between provincial and national institutions, which arbitrated the direction of public policy in the Litoral provinces.

$$
<\text { Congress }><\text { Railways }><\text { Nation State }><\text { Provinces }>
$$

* Una versión previa de este artículo fue presentada en el XXXIII Encuentro de Geohistoria Regional celebrado en septiembre de 2012; agradezco los comentarios realizados en esa oportunidad por los coordinadores Alicia Carlino y Enrique Schaller. Asimismo, agradezco los comentarios de los evaluadores de la revista.

* La investigación realizada para este artículo ha sido financiada por una beca de investigación doctoral inicial FONCyT.

* Magíster en Investigación Histórica. Profesora Asistente UNGS, bressanrv@gmail.com
} 


\section{Introducción}

En los últimos años se produjo un significativo avance en los estudios referentes a la vinculación entre el desarrollo ferroviario y la construcción del EstadoNación argentino en la segunda mitad del siglo XIX. Trabajos pioneros destacaron la importancia del establecimiento de este sistema de transporte para el fortalecimiento de la autoridad nacional y el desarrollo económico regional. De este modo, la construcción de los ferrocarriles tuvo un papel fundamental, ya que permitió incrementar el control político sobre las provincias tanto a través del desplazamiento más rápido de las fuerzas armadas como de la formación de alianzas con las elites de algunas regiones como Cuyo, la Pampa y Tucumán gracias al fomento de los vínculos económicos (Balán, 1978; Oszlak, 1997).

Distanciándose de estas miradas, investigaciones recientes han puesto en relieve que la conformación del Estado-Nación es resultado de la construcción de un conjunto de acuerdos y de instituciones que las propias elites provinciales establecieron a partir de las diversas experiencias vividas en el proceso de inserción en el nuevo escenario nacional (Bragoni y Míguez, 2010; Míguez, 2011). Asimismo, el análisis del desarrollo de los ferrocarriles ha sido considerado sumamente eficaz para observar el funcionamiento de las instituciones nacionales que se formaron y afianzaron en esta etapa como los cuerpos técnicos burocráticos y el Congreso Nacional (Palermo, 2006a y 2006b; Gómez, 2007; Gómez y Schvarzer, 2007). En este sentido, Silvana Palermo ha señalado que el Parlamento funcionó como "una caja de resonancia", donde las demandas de las provincias a través de sus legisladores tendían a amplificarse. Así, el debate entre los diversos intereses regionales traducían no sólo los reclamos provinciales si no que impulsaban políticas más amplias a las proyectadas originariamente.

El presente trabajo se nutre de estas propuestas, que privilegian el estudio al interior de los espacios provinciales, para pensar el rol del Congreso Nacional en la construcción de los ferrocarriles en las provincias de Corrientes y Entre Ríos y discutir la noción de cooptación para explicar las relaciones entre el gobierno nacional y las elites provinciales, formulada por los estudios ya clásicos, en el proceso de dotación de infraestructura durante la formación del Estado-Nación. Asimismo, también se toma distancia de las investigaciones centradas en analizar la poca rentabilidad de los ferrocarriles como una de las principales causas del desarrollo marginal de estas provincias dentro del proceso de construcción vial de la segunda mitad del siglo XIX (Scalabrini Ortiz, 1945; Rebuelto, 1994; López, 1994). Consideramos que el estudio de los proyectos ferroviarios para Corrientes y Entre Ríos, a partir del análisis de los debates parlamentarios, correspondencia particular y oficial de los funcionarios públicos y artículos periodísticos, es posible dar cuenta de las dinámicas y las prácticas políticas entre las instituciones nacionales y provinciales que dirimieron la dirección de las políticas públicas para estas provincias durante la etapa de formación del EstadoNación.

En la primera parte se analizan las características del la región oriental de Entre Ríos y Corrientes, donde se ubicaba la traza del primer ferrocarril planeado para 
estas provincias, y cuál fue la recepción a nivel local de esta propuesta. La segunda parte se centra especialmente en examinar las dinámicas de los debates parlamentarios a partir de los cuales se impulsaron los proyectos ferroviarios para estas provincias en las décadas de 1860 y 1870.

\section{El proyecto del Ferrocarril del Este al interior del litoral de los ríos}

La primera propuesta para construir un ferrocarril en las provincias de Corrientes y Entre Ríos se formó a principios de 1863, cuando el ingeniero William Mac Candlish, en asociación con George Knight y John Smith, presentó ante el Ministerio del Interior una solicitud para hacerse cargo de la construcción de un ferrocarril en la región del litoral ${ }^{1}$.

El trazado proyectado para el Ferrocarril del Este se extendía sobre la región oriental de Entre Ríos y Corrientes, el primer tramo corría paralelo al río Uruguay y conectaba Concordia con Monte Caseros, el segundo tramo se internaba en la provincia correntina desde este puerto hasta Mercedes. Resulta relevante señalar que la región donde se planeaba construir el ferrocarril había vivenciado un continúo crecimiento que se aceleró profundamente en las últimas décadas de la primera mitad del siglo XIX.

Entre 1820 y 1840 , correntinos y entrerrianos extendieron y consolidaron sus fronteras hacia la margen oriental. A partir de 1825, Corrientes buscó ampliar la frontera oriental más allá del río Miriñay e incorporar los territorios de la cuenca del Uruguay pertenecientes a las Misiones Occidentales. Con la anexión de esta región en 1830, quedó bajo el poder provincial la mayor parte de la planicie del Pay Ubre, comprendida entre los ríos Corrientes y el Uruguay, cuyas características físicas, con abundantes arroyos y praderas propiciaban el desarrollo ganadero (Poenitz, 1987; Schaller, 1995).

Durante estos años, en Entre Ríos recobró fuerza el proceso de colonización de la región oriental iniciado en la etapa colonial y suspendido en el período revolucionario. La franja que bordeaba el río Uruguay se transformó en una zona muy dinámica. Por una parte, en el área rural se desarrolló una serie de establecimientos dedicados a las actividades agrícola-ganaderas para el abastecimiento de los mercados locales y los del Atlántico. Por otra parte, en la ciudad de Concepción del Uruguay, se estableció un nutrido grupo de propietarios dedicados a la producción saladeril y al servicio de fletes terrestres y fluviales de alcance regional. Al norte de ésta, el puerto de Concordia, establecido en 1832, se convirtió en un nexo comercial entre el alto y el bajo río Uruguay, tanto para los productos exportados e importados hacia y desde los mercados de ultramar, como para los frutos regionales provenientes del Paraguay y Brasil (Poenitz, 1981; Schmit, 1999).

Asimismo, se debe tener en cuenta que en esta etapa también se forjó un estrecho vínculo entre el sudoeste de la provincia de Corrientes y la región oriental entrerriana. La división y aislamiento entre los departamentos sobre la costa del Paraná con los del

1 Memoria del Ministerio del Interior presentada al Congreso Nacional en 1864, Buenos Aires, Imprenta del Siglo, pp. 263-267. 
Bressan. El Congreso Nacional y los proyectos para la construcción...

Uruguay, generada por la dificultad de transitar a través de los bañados y esteros- a la cual se sumaba la falta de puentes para cruzar los ríos Corrientes, Batel y Santa Lucíafavoreció un mayor tráfico mercantil hacia Entre Ríos, tanto para abastecer los saladeros en la costa del Uruguay como para dar salida a las producciones a través del puerto de Concordia en lugar del de Goya sobre el río Paraná (Schaller, 1995; Schmit, 2003).

Por lo tanto, el trazado ferroviario designado para el Ferrocarril del Este, muestra claramente el objetivo de potenciar la actividad mercantil que ya se había gestado durante la primera mitad del siglo XIX, a través de la combinación del transporte terrestre y fluvial ${ }^{2}$.

El primer tramo del recorrido, correspondía a la sección no navegable del río Uruguay. Los productos que circulaban por la región eran transportados fluvialmente desde Misiones hasta Monte Caseros, donde la existencia del Salto Grande con una pendiente de 35 metros hasta el Salto Chico en la altura de Concordia tornaba intransitable este tramo del río. Por lo tanto, las mercaderías eran desembarcadas y llevadas por medio de carretas hasta Concordia, punto a partir del cual se podía navegar sin ninguna dificultad hacia los puertos del Plata. El segundo tramo, se internaba hasta el centro de la región sudeste de Corrientes, donde se habían establecido las estancias que abastecían los saladeros de Brasil y del oriente entrerriano.

De esta forma, la concreción de este proyecto prometía generar un gran impulso a la región, optimizando los transportes terrestres en combinación con los fluviales y superar las dificultades de navegación del Uruguay ${ }^{3}$. No obstante, las visiones acerca de los objetivos a alcanzar a partir de su emplazamiento no eran homogéneas.

Para los dirigentes entrerrianos, el Ferrocarril del Este se presentaba como la oportunidad de concretar- aunque en escala mucho menor- las ambiciones gestadas en años anteriores: convertir a los puertos en la margen del río Uruguay en los núcleos concentradores de un espacio mercantil que contactara toda la región del Alto Litoral y los mercados de ultramar ${ }^{4}$. Más aún, la puesta en marcha de esta obra no beneficiaría únicamente a Entre Ríos, como se expresaba en el diario oficial, sino a las dos provincias:

"Cien o ciento cincuenta mil cabezas de ganado en pie que pueden conducirse a Concordia, otra igual cantidad de cueros secos y las grasas correspondientes; la inmensa cantidad de yerba y maderas de que el ferrocarril va a ser conductor formando recién ese comercio en una escala difícil hoy de calcu-

\footnotetext{
2 Ver Anexo número 1.

3 En el mismo año que se sancionó la ley para la construcción del Ferrocarril del Este, el Congreso Nacional acordó una subvención mensual de ochocientos pesos durante tres años a la Sociedad Concordia para que realizara tres viajes mensuales en un buque de vapor entre los puertos de Federación y Santa María en el Alto Uruguay. Ver Congreso Nacional Cámara de Diputados Diario de Sesiones, sesión del 4 de julio de 1864, pp. 154-156.

${ }^{4}$ En la etapa de la Confederación se elaboraron diferentes proyectos con este objetivo pero que fueron imposibles de concretar. Sobre estos aspectos ver Schmit, Roberto. 2007. Los límites del progreso: expansión rural en los orígenes del capitalismo rioplatense. Buenos Aires, Siglo XXI, pp. 141-152.
} 
lar así como los retornos en mercaderías para una gran parte de la campaña de Corrientes, cuya población se irá aumentando considerablemente, [...] Al camino de fierro en Concordia no tardarán en seguirles otros y estas provincias cumplirán entonces su porvenir"5.

Entre los sectores dirigentes de la provincia de Corrientes, este proyecto respondía a los intereses de controlar, a través del desarrollo de vías de comunicación en la región oriental, los territorios misioneros y la explotación de sus recursos naturales (Buchbinder, 2004). Pero esta mirada no era compartida por algunos sectores que lo veían como un perjuicio para sus intereses. En este sentido, el Ferrocarril del Este convalidaba una dirección comercial que no beneficiaba a la provincia y que era sólo resultado de las dificultades para cruzar el río Corrientes. Así, en el diario La Esperanza de Goya se encontraba tanto las expresiones de rechazo a este proyecto como también el planteo acerca que el principal problema a resolver en materia de vías de comunicación no era la implementación de los modernos caminos de hierro sino la comunicación interna de la provincia a través del desarrollo de puentes y caminos. Perspectiva que se hallaba constantemente en varios periódicos correntinos a lo largo de las décadas de 1860 y $1870^{6}$.

Más allá de las diversas apreciaciones que suscitó éste ferrocarril, el proyecto iniciado por William Mac Candlish, en sociedad con la firma inglesa de Knight y Smith, contó con el respaldo del gobierno nacional el cual asumió los gastos derivados de los estudios requeridos para la construcción del ferrocarril y solicitó la colaboración de los gobiernos de Corrientes y Entre Ríos para que auxiliasen a los ingenieros convocados para aquella tarea ${ }^{7}$.

Sin embargo, todos los emprendimientos de este tipo conjugaban una gran inversión de capitales y un alto grado de incertidumbre con respecto a la rentabilidad futura que aportaría este medio de transporte.

Por este motivo, el impulso del desarrollo ferroviario en esta etapa se llevó a cabo por una combinación de capitales privados y la garantía del Estado nacional sobre las inversiones realizadas. En este aspecto, el Congreso Nacional desempeñó un rol clave para determinar qué proyectos contarían con este respaldo económico y cuáles no.

\section{Propuestas, impulsos y debates}

En 1864, cuando ya se habían realizado los estudios y planos, se sometió su aprobación en el Congreso Nacional. Los debates efectuados en ambas cámaras

5 El Uruguay, "Ferrocarril de Concordia", Concepción del Uruguay, 20 de octubre de 1864.

6 La Esperanza, "Un puente sobre el río Corrientes", 21 de junio de 1863.

7 Ver Carta del Ministro del Interior Guillermo Rawson a William Mac Candlish, Buenos Aires, 14 de abril de 1863 y carta del Ministro del Interior Guillermo Rawson a los gobernadores de las provincias de Entre Ríos y Corrientes, Buenos Aires, 21 de agosto de 1863, en Memoria del Ministerio del Interior presentada al Congreso Nacional en 1864, Op. cit., pp. 263-266. 
reflejaban el consenso de este emprendimiento entre la mayoría de los legisladores. Al interior del Senado, el representante de Santa Fe, José María Cullén, lideró su defensa en un extenso discurso cuyos principales argumentos podrían resumirse en dos tópicos. El primero se centraba en la experiencia de los contratistas en la construcción de ferrocarriles y la solidez de sus capitales. El segundo argumento subrayaba el carácter compensatorio que tendría la aprobación del proyecto para estas dos provincias "las que más contribuyeron a derribar el poder ingrato del General Rosas" y que colaboraban notablemente en la formación del Tesoro nacional ${ }^{8}$. De esta forma, se introducía en el debate la utilización del rol desempeñado por Entre Ríos y Corrientes durante la década de 1850 como un argumento central que permitía legitimar sus reclamos de dotación de infraestructura ante el Estado nacional.

Mientras que en la Cámara de Senadores el debate se articuló en torno a las funciones del Estado para impulsar la inclusión de todas las provincias en el camino del progreso, las discusiones en la Cámara de Diputados se orientaron en torno a los aspectos económicos del proyecto.

Dos puntos se mostraron relevantes al respecto, la extensión del trazado y la rentabilidad de la región en vinculación a las obligaciones que tendría el Estado para el pago de la garantía. El proyecto originario planeaba desarrollar un primer tramo de vías entre Concordia y Federación pero luego de los estudios practicados se consideró más ventajoso que la primera sección se extendiera hasta Monte Caseros, como explicaba a la cámara el diputado por Salta, Joaquín Bedoya:

"Los empresarios desde que se resuelven a emplear un fuerte capital en una obra de esta naturaleza, quieren que tenga desde el principio cierta magnitud para que les ofrezca conveniencia; y el gobierno, una vez que la Nación va a hacer el sacrificio de pagar la garantía ofrecida, quiere que este sacrificio de resultados más eficaces y benéficos. Así es que establecida la primera sección como estaba sólo quedaba obligada la empresa a construir 30 millas de camino. Es cierto que estas 30 millas resolverían el problema del Salto... pero era necesario algo más para ligar el comercio de Concordia con el de Monte Caseros"9.

Los datos presentados a la Comisión de Hacienda estimaban, en base a la cantidad de toneladas que se conducían en ese momento entre las dos localidades, que si la primera sección se extendía hasta Monte Caseros el pago en transporte ascendería a un millón doscientos mil pesos fuertes, suma que aumentaría una vez puesto en servicio

8 Congreso Nacional, Cámara de Senadores, Diario de Sesiones, (en adelante CNCSDS), sesión del 10 de septiembre de 1864, pp. 421-423.

9 CNCDDS, sesión del 1 de octubre de 1864, p. 887. 
el ferrocarril. Estas cifras alentaban la decisión de ofrecer la garantía del Estado y varios diputados consideraban que ésta solo tendría que ser pagada el primer año ${ }^{10}$.

La construcción del Ferrocarril del Este se autorizó a través de la ley número 120 , entre los artículos se destacaban ciertas condiciones favorables para los empresarios como el pago por cuarenta años de una garantía de un siete por ciento de interés anual sobre el capital empleado; la concesión sin cargo alguno de los terrenos necesarios para la línea férrea, estaciones, embarcaderos, galpones y depósitos y, por último, la posibilidad de importar sin gravamen alguno, por el lapso de cuarenta y ocho años, todos los materiales necesarios para la construcción y consumo del ferrocarril ${ }^{11}$.

Si bien el 11 de octubre de 1864, el gobierno nacional celebró el contrato con Tomás Finlayson, representante de los señores George Knight y John Smith, este quedó rescindido meses más tarde ya que los empresarios exigían a las provincias un uno por ciento adicional a la garantía ofrecida en el contrato o que cediesen a la empresa terrenos a los lados de la vía que correspondieran con ese valor ${ }^{12}$.

La situación en que se hallaba el fisco de ambas provincias impedía que asumiesen estas exigencias y el gobierno nacional declaró que no contraería más concesiones que las que se habían ofrecido ${ }^{13}$. El inicio de la Guerra del Paraguay determinó que la mayor parte de los recursos fiscales se destinaran a los insumos necesarios para el enfrentamiento bélico. Por lo tanto, la construcción del Ferrocarril del Este al igual que numerosos proyectos de infraestructura que se formularon a inicios de la presidencia de Mitre, quedaron relegados a años más tarde.

Sin embargo, hacia 1868, cuando el fin de la Guerra del Paraguay comenzaba a vislumbrarse como cercano, se generó un panorama propicio para renovar los planes de infraestructura que habían sido postergados frente a los gastos que demandaba la participación del gobierno argentino en la Triple Alianza. Fue en este contexto que se volvió a plantear al interior del Parlamento la posibilidad de construir el Ferrocarril del Este.

A fines de septiembre, el Senado formuló un proyecto para dotar a la región de Cuyo con un ramal vial que conectase a Mendoza con el ferrocarril que se construía entre Córdoba y Rosario. En su tratamiento en la Cámara de Diputados se presentaron dos propuestas, la primera autorizaba al Poder Ejecutivo a construir un ramal que partiría de Villanueva y se extendería hasta Mendoza, atravesando las localidades de Río Cuarto,

${ }^{10} \mathrm{El}$ incremento estimado por los legisladores era sumamente alto si se tiene en cuenta que ese mismo año la recaudación de las aduanas de Corrientes y Entre Ríos ascendió a 599.762,52 pesos fuertes. Ver: Memoria de Hacienda correspondiente al año 1864, Buenos Aires, 1865.

${ }^{11} C N C D D S$, Leyes sancionadas por el Congreso Nacional en 1864.

${ }^{12}$ Memoria del Ministerio del Interior presentada al Congreso Nacional en el año 1865, pp. 13- 14.

${ }^{13}$ Un análisis más detallado de las dificultades de los gobiernos provinciales para aceptar las nuevas exigencias en Bressan, Raquel. 2012. "La difícil materialización de las vías de comunicación en el litoral durante la formación del Estado-Nación (1860-1880)". En: Actas digitales del XXXII Encuentro de Geohistoria Regional. Instituto de Investigaciones Geohistóricas/Conicet/UNNE, Resistencia, pp. 625638. 
Bressan. El Congreso Nacional y los proyectos para la construcción...

Mercedes y San Luis. La segunda incluía, además del ramal previsto por el Senado, un ferrocarril desde Córdoba a Salta y otro desde Concordia a Mercedes ${ }^{14}$.

La construcción de ferrocarriles en el país contaba con la anuencia compartida acerca de las expectativas que las elites volcaban en el desarrollo de los caminos de hierro, como lo expresaba Francisco Civit:

"La construcción de caminos de fierro significa para nosotros asegurar la paz de la República, garantir la vida de sus habitantes y poner a los pueblos en comunicaciones muy frecuentes entre sí. Por consecuencia, me parece inútil esforzarme a demostrar a la Cámara, las conveniencias tanto políticas como económicas que reportarían al país una vez que se establezcan estos ferrocarriles" ${ }^{\prime 15}$.

Pero el desarrollo de los ferrocarriles y los beneficios adjudicados a él no podían ser otorgados por el estado nacional para todas las provincias en conjunto y simultáneamente. Por este motivo, el Congreso se convirtió en el principal espacio donde se debatió cómo serían otorgadas esas prioridades. Los argumentos en torno a qué proyecto debía contar con el apoyo de la cámara se articularon en base a las necesidades regionales. Francisco Civit, legislador por Mendoza, subrayaba los problemas de las provincias cuyanas para comunicarse y proveerse tanto con el litoral como con Chile, a lo que se sumaba la inseguridad al comercio por ser una zona de frontera. Cleto Aguirre, en defensa del proyecto en disidencia, señalaba que el objetivo de los ferrocarriles era poner a todas las provincias en condiciones de exportar sus productos y de introducir las mercancías para su consumo. A su voz se sumaba la del representante porteño Manuel Montes de Oca que destacaba que si bien las provincias de Entre Ríos y Corrientes contaban con la ventaja de las vías fluviales padecían las enormes dificultades de navegación del río Uruguay.

Además de exponer el grado de prioridades que el gobierno debía otorgar a cada provincia, consideramos importante detenernos en algunos aspectos sobre la construcción de ferrocarriles que se debatieron en estas sesiones y que dan cuenta de temáticas aún no resueltas en materia de obras ferroviarias en general.

Uno de ellos era la forma en que se obtendrían los capitales para estos proyectos. Este era un punto clave para el proyecto del Senado, ya que se consideraba que el Estado disponía de recursos para solventar a sólo uno de ellos. Así su primer artículo establecía que se designaría un 2 por ciento adicional a los derechos de exportación y un 5 por ciento a los de importación, con lo cual se esperaba obtener una suma superior a los dos millones de pesos anuales. Frente a esta disposición, el cordobés Luis Vélez señalaba que no sería posible utilizar estos montos porque ya habían sido afectados para la guerra

\footnotetext{
${ }^{14}$ El proyecto en disidencia fue presentado por los diputados Carlos Keen de Buenos Aires; Cleto Aguirre de Salta y Vicente Montero de Entre Ríos. Ver: $C N C D D S$, sesión del 5 de octubre de 1868.

${ }^{15}$ CNCDDS, sesión del 5 de octubre de 1868, p. 471.
} 
del Paraguay, por lo tanto era necesario solicitar un empréstito cuya garantía de pago sería este adicional. Carlos Keen presentó una opción diferente, ya que consideraba que las rentas generales, una vez finalizada la guerra del Paraguay, aportarían los capitales requeridos sin tener que recurrir a gravar aún más a la población con un aumento de los impuestos.

Un segundo tópico, en el que también emergieron posiciones encontradas, se adentraba en considerar si el Estado nacional debía ser constructor o accionista. Cleto Aguirre argumentaba que, en base a la experiencia de otros países como Inglaterra, Francia y Estados Unidos, los gastos de explotación eran menores en los ferrocarriles construidos por el gobierno que los realizados por empresas particulares. Por este motivo, el proyecto sancionado debía dotar al gobierno de "fondos suficientes para ponerlo en aptitud de hacerse constructor”.

En un análisis completamente opuesto, Carlos Keen subrayaba que los ferrocarriles del Estado, realizados en los países mencionados por el diputado de Salta, habían producido siempre malos resultados desde el punto de vista industrial y sólo habían servido a fines políticos. Subrayaba claramente su posición al declarar que otorgar la construcción de los ferrocarriles al Estado era cederle un poder mucho mayor al que ya poseía, en tanto disponía de los fondos del tesoro público y estaba al mando de la distribución y organización de la fuerza militar. Para otros diputados, en cambio, se debía dar amplia libertad al Ejecutivo para que fuese éste quién decidiera en cada caso que opción resultaba más conveniente en cada caso en particular.

Finalmente, se sancionó el proyecto de los diputados en disidencia respaldado por la concepción de que todas las regiones requerían el desarrollo de la red vial como elemento clave para potenciar la actividad económica de sus localidades, que el crecimiento de una región repercutiría inmediatamente en el resto del país y que este transporte, una vez puesto en funcionamiento, resarciría con creces las inversiones realizadas.

En este sentido, se debe tener en cuenta que las argumentaciones de los legisladores se inscribían dentro de un horizonte republicano acerca de las funciones del Congreso que ya se había delineado durante la etapa de la Confederación y se consolidó en las décadas siguientes: las leyes debían procurar un proceso de transformación y modernización socioeconómica que garantizaran el bienestar general. En este contexto, las demandas de obras de infraestructura a las instituciones nacionales no se asentaban en los intereses locales sino que se legitimaban en la necesidad de afianzar la inclusión de las provincias en el territorio nacional (Lanteri, 2011; Palermo, 2006a).

Asimismo, las discusiones relevadas en los párrafos anteriores, excedían los reclamos provinciales para obtener los caminos de hierro y se insertaban, en los albores del desarrollo ferroviario, en la formulación de fundamentos básicos y en la dirección que las políticas públicas debían acordar en materia vial. En circunstancias como las de las primeras décadas de la segunda mitad de siglo XIX, cuando la experiencia ferroviaria era muy incipiente, estos debates al interior del Congreso Nacional muestran cómo se fueron definiendo los límites que marcarían las pautas de acción del Estado y 
Bressan. El Congreso Nacional y los proyectos para la construcción...

que luego se condensarían en la ley general sobre la Administración y Dirección de los Ferrocarriles sancionada en septiembre de 1872.

Si nos remitimos al Ferrocarril del Este en particular, observamos un notable cambio en el rol que tuvo el Congreso en la sanción del proyecto en 1864 con respecto al tratado en las sesiones de 1868. Como se planteó en párrafos anteriores, en 1864 el Congreso se limitó a autorizar una propuesta que ya se había diseñado previamente a partir de negociaciones entre los contratistas y el Ejecutivo Nacional. Contrariamente, en 1868 la propuesta de llevar a cabo su construcción surgió, como señala Palermo, de impulsos originados por el propio funcionamiento de la Cámara de Diputados (Palermo, 2006a).

De esta forma, el Parlamento se ubica en los primeros pasos que recorre la trayectoria de la construcción del Ferrocarril del Este a partir de 1868. Esta posición del Congreso como ámbito dónde se impulsó el proyecto se observa en los pasos siguientes realizados en su construcción. La ley sancionada establecía que cuando el Estado nacional contase con los fondos públicos necesarios avalaría la obra con una garantía del 7 por ciento, por este motivo las acciones se centraron en buscar a los contratistas que quisiesen hacerse cargo de este proyecto y buscar el apoyo estatal para concretarlo.

En esta segunda etapa, Benjamín Victorica, senador por Entre Ríos, tuvo una activa participación. Se puso en contacto con Luis Varela y con el Ministro del Interior, Dalmacio Vélez Sarsfield, para que por su intermedio se llevaran a cabo nuevos estudios a cargo de la Oficina de Ingenieros Nacionales y para que el proyecto no quedase relegado frente a los múltiples compromisos asumidos por el gobierno nacional. Asimismo, solicitó a Ladislao Rodríguez, administrador de rentas nacionales de Concordia, que elaborase un informe con los frutos exportados e importados en esta plaza y establecer en base a estos las utilidades que reportaría el tráfico comercial con el desarrollo del ferrocarril ${ }^{16}$. Un año más tarde estas acciones dieron sus frutos $\mathrm{y}$, el 12 de agosto de 1869, Pablo Montravel celebró un contrato con el gobierno nacional, asumiendo su construcción con capitales propios y en consorcio con otros accionistas por un valor de 10.000 libras por milla (Duarte, 1965).

Una dinámica similar a la del Ferrocarril del Este se observa en la formulación de un proyecto para extender el ferrocarril de Mercedes a Corrientes. En 1872 el senador por San Juan, José María del Carril, presentó un ambicioso proyecto que buscaba conectar las provincias de Cuyo con los mercados del norte, de Buenos Aires y de Chile a través de cuatro líneas establecidas entre las siguientes localidades: desde Buenos Aires hasta Mendoza; desde Mendoza y San Juan hasta San Felipe de los Andes; desde Tucumán hasta San Juan y desde Tucumán hasta Jujuy ${ }^{17}$.

\footnotetext{
${ }^{16}$ Archivo General de la Nación, Fondo Benjamín Victorica, leg. 15, Carta de Justo José de Urquiza a Benjamín Victorica, San José, 5 de noviembre de 1868; Carta de Benjamín Victorica a José Sagastume, Buenos Aires, 8 de noviembre de 1868; Cartas de Luis Varela a Benjamín Victorica, Buenos Aires, 13 de noviembre de 1868, 24 de noviembre de 1868, 7 de diciembre de 1868, 9 de diciembre de 1868; Carta de Dalmacio Vélez Sarsfield a Benjamín Victorica, Buenos Aires, 5 de diciembre de 1812, Carta de Ladislao Rodríguez a Benjamín Victorica, Concordia, 9 de diciembre de 1868.

${ }^{17}$ CNCSDS, sesión del 5 de septiembre de 1872 .
} 
No obstante, en el desarrollo del debate, el senador de Entre Ríos, Teófilo García, planteó la posibilidad de incluir también en el proyecto el ramal entre Mercedes y Corrientes, atravesando las localidades de Saladas y San Roque, el cual sería posible si la línea de cuyo en lugar de partir de Buenos Aires lo hiciera de la Villa de Mercedes en San Luís.

Esto generó una fuerte oposición en cómo se establecían los trazados ferroviarios garantizados por el Estado Nacional en relación con los aportes generados por las provincias y que se expresaba en la voz de José María del Carril:

"Si cuando la Nación trataba de hacer un gran sacrificio, echando sobre su tesoro la obligación de una erogación de más de tres millones y medio de pesos fuertes anuales, para dotar a la república de la red de ferrocarriles que se proyectaba, por razones de economía se había de excluir a Buenos Aires, que contribuía con más de los dos tercios de la renta, de los grandes beneficios que se preveían, valía más rechazar el proyecto y tirarlo debajo de la mesa”"

Al igual que el peso de las rentas que el litoral aportaba a la Nación había sido uno de los factores en la defensa de la construcción del Ferrocarril del Este en 1864, el mismo planteo se generaba para respaldar el trazado de Buenos Aires a Mendoza.

Debemos tener en cuenta que los ingresos provenientes de las aduanas representaban el mayor porcentaje de la recaudación nacional y habían constituido hasta su nacionalización, la mayor fuerte de ingresos para los erarios provinciales (Schaller, 2002; Schmit, 2007). Por este motivo, los planteos con respecto a la otorgación del siete por ciento de garantía para la construcción de los ferrocarriles se vinculaban, por una parte, a un reclamo de las provincias como una compensación por los ingresos que habían dejado de percibir y, por otro, a cómo la inversión estatal produciría, una vez finalizadas las obras, mayores aportes al Tesoro.

Al observar las cifras registradas de los aportes provinciales a las rentas generales de la nación, se reafirma el argumento del senador Carril de no excluir a Buenos Aires. En ese año las rentas de la nación ascendieron a 12.700.298,82 pesos fuertes de los cuales el 77,05\% habían sido aportados por la provincia porteña. Ahora bien, las cantidades aportadas por Entre Ríos y Corrientes, si bien eran marcadamente inferiores, con un $6,30 \%$ y $1,43 \%$ respectivamente, resultaban significativamente mayores que las aportadas por las otras localidades en las cuales se emplazarían los ramales previstos por el proyecto original: Mendoza 0,87 , San Juan 0,62 y Jujuy $0,09^{19}$.

\footnotetext{
${ }^{18}$ CNCSDS, sesión del 27 de septiembre de 1872.

${ }^{19}$ Los datos son de elaboración propia en base a Memoria del Ministerio de Hacienda presentada al Congreso Nacional en 1872, Buenos Aires, Imprenta de la Unión, 1872.
} 
Por lo tanto, el mismo argumento para no excluir a Buenos Aires también se tornaba aplicable para incluir la prolongación del Ferrocarril del Este. Al pensar en la disparidad de recursos económicos aportados por las provincias y su capacidad de negociación en el nuevo escenario nacional, la historiografía abocada a esta etapa planteó que el Senado, con una representación igualitaria para todas las provincias, encarnó un contrabalanceo del poder a favor de las provincias del interior por sobre las del litoral (Ozlak, 1997; Gorostegui de Torres, 2000).

Sin embargo, observamos que, si bien la propuesta de García fue resistida, los senadores de Entre Ríos y Corrientes- que se encontraban en desventaja numéricamentebuscaron demostrar las ventajas de este proyecto y alcanzar el voto de la mayoría. Así, Wenceslao Colodrero y Eusebio Torrent, ofrecieron un nutrido panorama de los beneficios de la realización del ramal entre Mercedes y Corrientes, las cuales se centraban principalmente en los aportes que se obtendrían no sólo para Corrientes y Entre Ríos, sino para las provincias del norte también, una vez que se realizara la navegación del Bermejo y las producciones del noroeste argentino tuvieran salida a través del puerto de Concordia. Además, la ejecución de este ramal haría posible la definitiva comunicación entre la capital de la provincia y los departamentos al sur, por lo tanto, ya no sería necesaria la realización de los puentes sobre los ríos Batel, Santa Lucía y Corrientes que también se iban a construir con fondos nacionales ${ }^{20}$.

Tanto en el Senado como en la Cámara de diputados se aprobó el proyecto con las modificaciones establecidas por los senadores García, Torrent y Colodrero, a través de la ley 583 del 5 de noviembre de $1872^{21}$. Bajo sus bases se celebró un contrato en 1874 con la compañía Furnes para llevar a cabo su construcción con una garantía del 7\% durante 20 años (López, 1994).

La sanción de esta ley estableció un precedente que posibilitó la aprobación de una propuesta para la extensión del Ferrocarril del Este hacia el sur, desde Concordia hasta Gualeguaychú. Esta línea ya había sido autorizada por el gobierno de la provincia de Entre Ríos y los legisladores buscaban obtener en el Congreso Nacional la concesión de una garantía sobre el capital invertido para la construcción.

La propuesta encontró una férrea oposición nuevamente en la Cámara de Senadores ya que se consideraba que el Congreso no podía avalar el pago de garantías para trenes de explotación local, porque los beneficios obtenidos serían solamente para la provincia y no para la Nación. Así lo indicaba el informe presentado por el senador de Jujuy, Placido Bustamante, y agregaba que la concesión de una garantía a un tren provincial seria perjudicial a la recaudación:

"Si hoy se concediese la garantía pedida por el gobierno de Entre Ríos, mañana no sería posible negarla a otras provincias del interior que colocadas en peores condiciones tendrían más poderosas razones para ser atendidas"22.

\footnotetext{
${ }^{20}$ CNCSDS, sesión del 28 de septiembre de 1872, pp. 274-279.

${ }^{21} C N C D D S$, Leyes sancionadas por el Congreso Nacional en 1872.

${ }^{22} C N C D D S$, sesión del 7 de junio de 1873, p. 128.
} 
En la defensa de ese proyecto, Nicasio Oroño, representante santafesino, establecía que este se encuadraba dentro de las mismas condiciones que el ramal que se había aprobado el año anterior entre Mercedes y Corrientes, en tanto que si bien eran líneas que se extendían únicamente en el espacio provincial, atraían las conexiones con otros mercados, y así como la de Corrientes se vincularía con el noroeste, la de Concepción del Uruguay lo haría con los mercados de la República Oriental.

Otro de los argumentos esgrimidos, era la obligación que tenía la Nación de acordar una protección a cada una de las provincias que solicitasen la ayuda del gobierno para impulsar las vías férreas en sus territorios, porque por fuera de Buenos Aires era difícil que alguna estuviese en condiciones de impulsar por sí sola estos proyectos ${ }^{23}$.

En la discusión que tuvo lugar en la cámara de diputados, los representantes por Entre Ríos, Ángel Elías y Onésimo Leguizamón, apelaban a una gama de razones que pueden ser resumidas en tres ejes. En primer lugar, la planificación de una línea férrea, por parte del país vecino de Uruguay, desde el Salto hasta Uruguayana para atraer el comercio brasileño de la región de las misiones, se convertiría en un fuerte competidor del ferrocarril que ya se estaba construyendo sobre la margen occidental del río Uruguay y que contaba con la garantía estatal. En segundo lugar, el trazado de la línea uniría departamentos de gran importancia: Concordia con Colón, donde se ubicaba "una de las más importantes colonias de la República Argentina"; Concepción del Uruguay, capital provincial que poseía grandes saladeros a su alrededor y, por último, Gualeguaychú "una de las más prósperas y florecientes" localidades de Entre Ríos. Un último argumento se refería a los levantamientos ocurridos en la provincia:

"Entre Ríos es tal vez la única provincia de la República donde por desgracia se levanta la bandera de la segregación, condenada por todos los buenos argentinos.

Si el ferrocarril es un vínculo de civilización y nacionalidad, si el progreso es una cadena aprisionadora, aprisionemos a este pueblo con esa cadena de progreso, una vez que trata de emanciparse por medio de la rebelión.

Estas consideraciones deben pesar mucho en el ánimo de los señores diputados: conviene ligar ese sentimiento extraviado de una parte del pueblo de Entre Ríos con empresas que atestigüen de una manera eficaz la protección eficaz que la Nación dispensa al progreso y adelanto de aquella provincia"24.

De esta forma, las palabras de Onésimo Leguizamón buscaban transformar las consecuencias negativas que habían tenido las rebeliones de López Jordán para el desenvolvimiento de los dos ferrocarriles existentes en la provincia y que, probablemente, desalentaban el apoyo para desarrollar nuevos planes en materia ferroviaria ${ }^{25}$.

\footnotetext{
${ }^{23}$ CNCSDS, sesión del 7 de junio de 1873, pp. 128-136.

${ }^{24}$ CNCDDS, sesión del 26 de septiembre de 1873, pp. 1245-1250.

${ }^{25}$ Sobre las consecuencias de las rebeliones de López Jordán en la construcción del Ferrocarril del Este ver
} 
Bressan. El Congreso Nacional y los proyectos para la construcción...

A pesar de los argumentos en oposición, principalmente en el Senado, la propuesta llevada adelante por los legisladores entrerrianos fue aprobada y el Congreso Nacional otorgó la garantía del 7 por ciento sobre el capital invertido para esta nueva prolongación el 30 de septiembre de $1873^{26}$.

Durante las décadas de 1860 y 1870, a partir de la actividad en el Congreso Nacional se promovió un significativo trazado vial para la región del litoral. Así, el Ferrocarril del Este en articulación con los dos ramales hacia el norte y el sur, tenía como estaciones a Gualeguaychú y Corrientes sobre la costa del Paraná; atravesaba toda la franja más dinámica de Entre Ríos y Corrientes sobre la costa del UruguayConcepción del Uruguay, Colón, Concordia, Federación y Monte Caseros- y unía la región sureste de Corrientes con la capital a través de Mercedes, San Roque y Saladas.

Más aún, a través del accionar en el Congreso, no sólo los legisladores habían conseguido que se siguieran impulsando estos proyectos a pesar de la conflictiva situación bélica existente en sus territorios, sino que lograron -para dos líneas que se extendían únicamente al interior de las provincias- las mismas concesiones que se otorgaba a los ferrocarriles nacionales. Este es un punto que consideramos significativo en tanto que la garantía del siete por ciento sobre el capital invertido, sólo se otorgaba a vías férreas que unían localidades de distintas provincias. El único caso en que se obtuvo para un ferrocarril provincial en esta etapa, había sido para el de Buenos Aires a Campana, porque se consideraba que, de acuerdo al volumen de producción de la región, el Estado nacional no tendría que pagar la garantía por un lapso mayor a un año.

En suma, el Congreso Nacional constituyó un espacio clave en el cual las provincias de Corrientes y Entre Ríos pudieron impulsar proyectos ferroviarios particulares que contasen con respaldo del gobierno nacional dentro de las demandas en conjunto del resto de las provincias.

En este sentido, consideramos que las distintas posturas expresadas en los debates parlamentarios efectuados en 1868, 1872 y 1873 nos permiten pensar en una dinámica mucho más compleja que una relación de cooptación que buscaba tejer alianzas entre los sectores dominantes provinciales para consolidar el sistema de dominación impuesto en el orden nacional (Oszlak, 1997).

Si bien los subsidios y el empleo público constituían mecanismos de cooptación que el Estado nacional podía utilizar discrecionalmente para formular consenso entre los sectores sociales dominantes, la aprobación de proyectos- como los de desarrollo ferroviario- no podía ser manipulada unilateralmente. La obligación de que estos proyectos fuesen aprobados por el Congreso Nacional los sometía a la evaluación de voces plurales que se manifestaban con distinto grado de concordancia u oposición. Por lo tanto, la toma de decisiones acerca de qué compromisos- y qué sectores o provincias serían favorecidos- no quedaba limitado a un restringido grupo, si no a la capacidad de acción de los legisladores que buscaban la aprobación a través del voto de la mayoría.

Duarte, María Amalia. 1965. "Los ferrocarriles de Entre Ríos durante la presidencia de Sarmiento", En:

Trabajos y Comunicaciones, No 13, La Plata, Universidad Nacional de La Plata. pp. 49-74.

${ }^{26} C N C D D S$, Leyes sancionadas por el Congreso Nacional en 1872. 
Resulta pertinente, entonces, ampliar brevemente el perfil de algunos de los legisladores del litoral de los ríos que impulsaron estas propuestas en el Parlamento. La mayor parte de ellos se hallaban insertos dentro del circulo urquicista y ya habían tenido una importante participación en el Congreso de Paraná como Wenceslao Colodrero, Benjamín Victorica, y Ángel Elías, experiencia que les permitió forjar relaciones con los representantes de otras provincias que continuaron su rol legislativo durante las décadas de 1860 y 1870 (Lanteri, 2011).

A su vez, legisladores de una generación más joven como Onésimo Leguizamón y Juan Eusebio Torrent, comenzaron su actividad política primero a nivel local pero también lograron crear una serie de vínculos más allá de su provincia natal en forma previa a su ingreso al Congreso. Así Leguizamón, quien había estructurado su vida política bajo la protección del urquicismo, se trasladó a la ciudad porteña luego de la muerte de Justo José de Urquiza y comenzó a trabajar como redactor en La Prensa, cuyo dueño y colaboradores habían cursado con él los estudios de abogacía en la Universidad de Buenos Aires. Además, reanudó relaciones con sus excompañeros del Colegio del Uruguay como Eduardo Wilde, Julio Argentino Roca y Victorino de la Plaza y comenzó a establecer estrechos lazos con los círculos ligados a Nicolás Avellaneda, quien lo nombró Ministro de Justicia, Culto e Instrucción Pública en 1874 (Vanossi, 2004).

La influencia de Torrent en la vida política correntina se registró en forma significativa a partir de su participación en la revolución liberal que derrocó al gobernador José María Rolón en 1861. La gestión de Torrent fue clave para obtener el apoyo de Mitre a favor de la facción liberal y las entrevistas en Buenos Aires del joven correntino con el presidente se tradujeron en una perdurable amistad que condujo a su nombramiento como Ministro Plenipotenciario en Brasil en el año 1866 y, más tarde, en su elección como vicepresidente en la campaña mitrista de 1874 (Palma, 1941).

Si bien consideramos que los legisladores hallaron en el Congreso Nacional un ámbito privilegiado para presentar los reclamos provinciales y utilizaron sus vínculos y contactos para lograr una adhesión que respaldara sus propuestas, al ubicar estos proyectos en esos espacios locales, se observa que las propuestas que ingresaban al recinto parlamentario no contaban con un consenso unánime. Ya habíamos señalado al principio de este artículo que el proyecto del Ferrocarril del Este no contaba con la adhesión de algunos departamentos al oeste de Corrientes, y principalmente Goya, que consideraban prioritario elaborar propuestas que fomentaran la articulación al interior de la provincia en lugar de profundizar la ya existente con Entre Ríos.

La instalación del Ferrocarril del Este y sus extensiones hacia el norte y el sur fueron los únicos proyectos ferroviarios para Corrientes y Entre Ríos discutidos en el Congreso Nacional en las décadas de 1860 y 1870, pero no fueron los únicos que alcanzaron cierto margen de desarrollo a nivel local y que buscaban ser considerados en las cámaras legislativas ${ }^{27}$.

\footnotetext{
${ }^{27}$ El ferrocarril "Primer Entrerriano", en el departamento de Gualeguay, también fue objeto de discusión en el Congreso Nacional pero no como un proyecto a realizar como los que hemos analizado en este artículo sino debido a su compra por parte del Estado Nacional, por lo cual no ha sido incluido en estas páginas.
} 
Bressan. El Congreso Nacional y los proyectos para la construcción...

En 1864, los vecinos de Paraná formaron una asociación presidida por Eusebio Ocampo- diputado nacional y también con una experiencia legislativa iniciada en el Congreso de Paraná que lo ligó al círculo urquicista- para construir una línea férrea entre esa ciudad y Nogoyá. La asociación logró que su proyecto fuese respaldado por el gobierno nacional, el cual comisionó al ingeniero Neville Mortimer para que realizara los estudios necesarios y el plano del trazado vial. Con los positivos informes finales las autoridades departamentales buscaron promover el proyecto a través de la prensa local pero el mismo no obtuvo el respaldo de los funcionarios provinciales y no alcanzó el recinto parlamentario ${ }^{28}$.

A su vez, el proyecto de extender el Ferrocarril del Este desde Mercedes a Corrientes se encontró con dos posturas diferenciadas. Por una parte, uno de sus principales promotores, el diputado Agustín P. Justo, concebía que el proyecto permitiría solucionar los problemas de comunicación interna de la provincia y, por este motivo, debía ser respaldado por el gobierno provincial a partir de ciertas concesiones que allanarían su debate en el Congreso Nacional, como la sesión gratuita de tierras para las vías y las estaciones ${ }^{29}$. Por otra parte, se encontraban los detractores de esta propuesta, entre quienes se destacaban los escritores del diario La Fusión, Manuel Derqui, Mariano Castellanos y Genaro Figueroa, quienes argentaban la necesidad de llevar a cabo otro tipo de obras que permitieran el enriquecimiento de los departamentos de la margen del Paraná y no aquellas destinadas a fomentar más aún la articulación comercial con los puertos del oriente entrerriano ${ }^{30}$. Por este motivo, buscaron promover la construcción de un ferrocarril que uniese los departamentos de Caa Cati, Mburucuyá, Saladas y Bella Vista. Sin embargo, fue el proyecto de Justo y no este el que concitó el favor gubernamental $^{31}$.

Ninguno de estas propuestas traspasaron los límites provinciales en el camino hacia una posible discusión al interior del Congreso Nacional, pero estos "otros proyectos” nos permiten ver desde otro ángulo las interacciones entre los niveles nacionales y provinciales. Al interior del Parlamento los legisladores buscaban impulsar proyectos que beneficiarían a sus provincias, por lo tanto, podría decirse que se establecía una competencia- y una búsqueda de alianzas- a nivel interprovincial. Sin embargo,

\footnotetext{
${ }^{28}$ Carta de Eusebio Ocampo al Ministro del Interior, Guillermo Rawson, Paraná, 12 de noviembre de 1864, en Memoria del Ministerio del Interior presentada al Congreso Nacional en el año 1866, Op. cit., pp. 144-147; Archivo General de Entre Ríos, Fondo Gobierno, Serie 12, Jefaturas de Policía por Departamento, Subserie A "Jefatura de Paraná", caja 4, leg. 3, Carta de Eusebio Ocampo al Jefe Político de Paraná, Domingo Comás Paraná, 8 de febrero de 1865; Carta de Eusebio Ocampo al Ministro General de Gobierno, Paraná 9 de febrero de 1865; Memoria del departamento de Paraná, presentada al Ministerio General de Gobierno, Paraná, 15 de febrero de 1865.

${ }^{29}$ Archivo General de la Provincia de Corrientes, Registro Oficial, Carta de Desiderio Onieva a Agustín Justo, Corrientes, 23 de agosto de 1871. La Esperanza, "Un proyecto para Corrientes", 7 de agosto de 1871.

${ }^{30}$ El diario La Fusión fue creado por una fracción del partido liberal contraria al gobierno de Santiago Baibiene. Ver Mantilla, Florencio. 2007. Bibliografia periodística de la provincia de Corrientes (1887). Corrientes, Amerindia.

${ }^{31}$ La Fusión, "Proyecto de Ferrocarril", 9 de agosto de 1871, 11 de agosto de 1871, "El empréstito", 6 de septiembre de 1871; "El crédito de la provincia", 8 de septiembre de 1871.
} 
estos proyectos no reflejaban posturas homogéneas al interior de las provincias, por el contrario, en estos espacios convivían diversos actores que formaban parte de la articulación entre los distintos ámbitos de poder y desde los cuales se llevó a cabo un proceso de interacción y disputa constante.

En este sentido, las propuestas de implementar ramales ferroviarios en la región occidental de las provincias de Corrientes y Entre Ríos, que conectaran a los departamentos interiores con los puertos del Paraná, no alcanzaron un consenso a nivel local que les facultara avanzar hacia una instancia de discusión a nivel nacional como los proyectos centrados en articular las zonas productivas con los puertos en el río Uruguay. Incluso, aquella propuestas, como la del ferrocarril entre Nogoyá y Paraná, que habían obtenido un respaldo del Ejecutivo Nacional y que entre sus principales promotores se encontraba un diputado del Congreso Nacional, Eusebio Ocampo, no lograron traspasar los limites provinciales y ser objeto de discusión en el Parlamento.

Debemos tener en cuenta, como se describió al principio de este artículo, que estas diferentes propuestas respondían a una articulación regional que se había consolidado a mitad del siglo XIX donde las zonas más dinámicas a nivel económico se encontraban en la margen oriental de ambas provincias dedicada a la actividad pecuaria y a la utilización de los puertos entrerrianos de la costa del Uruguay para dar salida a estas producciones. A su vez, esta región tenía serias dificultades de comunicación con el espacio occidental. El avance y las reticencias sobre los proyectos para la región oriental y occidental deben comprenderse, además, dentro del marco de los conflictos políticos a nivel local. Como ha planteado Buchbinder, los grupos dirigentes correntinos asentados en la capital tenían serias dificultades para controlar a los jefes departamentales del sur. Si bien ambos conformaron una coalición que promovió la revolución liberal de 1861, esta alianza era sumamente frágil e inestable donde los caudillos sureños imponían constantemente sus condiciones a la dirigencia central correntina (Buchbinder, 2004). Por lo tanto, las obras destinadas a la región oriental podían ser visualizadas como una herramienta que aumentaba aún más la hegemonía de los grupos políticos de los departamentos del sur y reforzaba a su vez los lazos con el urquicismo, principalmente de jefes militares como Nicanor Cáceres, que no sólo operaba en calidad de estanciero en los puertos orientales entrerrianos si no que, además, mantenían fluidos contactos con el gobernador entrerriano en torno a las operaciones militares en la frontera entre ambas provincias ${ }^{32}$.

Consideramos, entonces, que el análisis desarrollado acerca de los proyectos ferroviarios para Entre Ríos y Corrientes contribuye a profundizar aquellas miradas

\footnotetext{
${ }^{32}$ Con respecto a este último argumento ver Archivo General de la Nación, Fondo Justo José de Urquiza, leg. 1765, Carta de Nicanor Cáceres a Justo José de Urquiza, Paraíso, 26 y 27 de febrero de 1868; leg. 1769, Carta de Nicanor Cáceres a Justo José de Urquiza, Paraíso, 23, 26 y 30 de mayo de 1868 y leg. 1771, Carta de Nicanor Cáceres a Justo José de Urquiza, Paraíso, 12 de julio de 1868. Debemos señalar que en 1871, cuando se propone la construcción del ferrocarril para los departamentos del noroeste correntino, las relaciones políticas entre el sur correntino y el gobernador entrerriano se modificaron profundamente luego de la Revolución de 1868 y el asesinato de Urquiza en 1870, pero este es un tema que aún debe ser indagado en profundidad.
} 
Bressan. El Congreso Nacional y los proyectos para la construcción...

que plantean que la construcción del Estado a nivel nacional no es sólo producto de "progresivas y simultáneas penetraciones en las provincias", si no que el proceso de centralización del poder resultó tributario de dos dinámicas convergentes: la provincial y la nacional. La presencia de la nación en las provincias debe ser comprendida teniendo en cuenta el conjunto de acuerdos y de instituciones que las propias elites provinciales establecieron a partir de las diversas experiencias que se desencadenaron y en el cual prevalecieron intereses de naturaleza variada, aunque sujetos a mecanismos e instrumentos simultáneos implementados tanto por los poderes locales como por los representantes o comisionados del poder central (Bragoni y Míguez, 2010).

Por este motivo, para poder reconstruir cabalmente cuáles fueron las dinámicas y las prácticas políticas entre las instituciones nacionales y provinciales es necesario profundizar también cuáles fueron las interacciones llevadas a cabo por los grupos dirigentes locales en las provincias de Corrientes y Entre Ríos durante la etapa de formación del Estado-Nación.

\section{Conclusiones}

El desarrollo de un sistema ferroviario dentro del proceso de construcción del Estado nacional ha sido considerado como un elemento clave- junto con otras obras de infraestructura como los puentes y caminos- que permitió incorporar diversas regiones dentro de un mercado de producción nacional y extender las bases de apoyo social al Estado en formación (Oszlak, 1997). En este sentido, se ha subrayado que las burguesías del interior, principalmente la azucarera de Tucumán y la vitivinícola de Mendoza, obtuvieron la construcción de ramales viales debido a su capacidad de negociación en un contexto donde el gobierno nacional buscaba sentar las bases de su poder en alianza con estos grupos y consolidar su poder en esa región (Balán, 1978).

Desde esta mirada, la escasa extensión que alcanzaron las vías férreas de Entre Ríos y Corrientes, parecería mostrar un rol marginal de estas provincias litoraleñas dentro del conjunto de obras llevadas a cabo en esta etapa, posiblemente como resultado de una escasa capacidad de negociación de sus dirigencias en el contexto de construcción del Estado-Nación ${ }^{33}$. Sin embargo, este trabajo, al analizar los proyectos y no los resultados concretos, muestra un panorama muy diferente.

Corrientes y Entre Ríos, a través del accionar de sus legisladores, obtuvieron negociaciones muy exitosas logrando para sus proyectos concesiones similares a las otorgadas a los ferrocarriles del Norte y de Cuyo. Más aún alcanzaron que se respaldara con una garantía estatal la construcción de ramales que unían localidades internas de las

\footnotetext{
${ }^{33} \mathrm{Al}$ finalizar la década de 1870 , se podría establecer que el trazado vial se hallaba distribuido en tres grupos: el interior, con el Ferrocarril Central Argentino (desde Rosario a Córdoba), el Andino (desde Córdoba a Mendoza y San Juan) y el Central Norte (de Córdoba a Tucumán) tenía una extensión en conjunto de 1.293 kilómetros; la provincia de Buenos Aires que contaba con 1.127 kilómetros y por último, las provincias de Corrientes y Entre Ríos en la región del litoral de los ríos sólo alcanzaban una prolongación 170 kilómetros de vías férreas. Ver Memoria del Ministerio del Interior presentada al Congreso Nacional de 1881, Buenos Aires, Establecimiento Tipográfico de La Pampa, 1881, p. 22.
} 
provincias en un contexto en donde se había afianzado que la intervención del Estado nacional se dirigiría a obras de infraestructura que comunicasen a las provincias y no a emprendimientos que tenían como objetivos beneficios "puramente locales"34.

El Congreso Nacional era una institución clave para promover estas propuestas pero queremos replantear dos aspectos que consideramos centrales de las dinámicas desarrolladas en esta institución. Por una parte, lejos de funcionar como una instancia donde los representantes provinciales convalidan la política del Ejecutivo nacional en su búsqueda de alianzas con las elites provinciales las cuales permitiría granjear al Estado en construcción la centralización del poder, el Parlamento promovía el planteo de posturas divergentes que a través del debate construyeron en esta etapa las directivas a seguir en materia de obra pública.

Como señala Gianfranco Poggi, la controversia y el conflicto en forma regulada componen los rasgos esenciales del Estado moderno y el Parlamento es el ámbito por excelencia de discusión y de procesos vitales para la toma de decisiones. En este sentido, el Congreso no transmite simplemente los impulsos que se originan en otras partes sino que es productor también de estos impulsos (Poggi, 1997).

El rol de productor se observa en las sesiones de 1868,1872 y 1873 , cuando en el marco de la discusión de planes de infraestructura para otras regiones del país se formularon y aprobaron las propuestas ferroviarias para Corrientes y Entre Ríos. A su vez, en una etapa donde los cuadros técnicos estaban en formación, los legisladores asumieron la función de determinar los trazados, el tipo de trocha y el grado de intervención del Estado como constructor o como garante, sin que las decisiones tomadas en su seno fueran disputadas por las dependencias estatales especializadas en cuestiones de obra pública.

Por otra parte, la estructura del Parlamento, con una representación sobre una base demográfica para la Cámara de Diputados y una igualitaria para el Senado, fue visualizada por la historiografía como un contrabalanceo del poder a favor de las provincias del interior que colocaba a las provincias de Corrientes y Entre Ríos en una clara desventaja con respecto a las provincias del interior para los debates en este último recinto (Gorostegui, 2000). Estas desventajas se profundizaban en las discusiones referentes a dirimir prioridades en materia de infraestructura, como las analizadas en este artículo, donde las provincias del interior buscaban vincular sus producciones en el litoral, pero especialmente con Rosario y Buenos Aires.

A pesar de estas desventajas, los legisladores entrerrianos y correntinos lograron que sus propuestas fueran aprobadas en esta Cámara. En el presente artículo hemos analizado los argumentos que utilizaron para defender sus propuestas, pero creemos que, como una practica usual en este recinto, estos sirvieron tanto para legitimarlas como para convencer a legisladores que no habían tomado una postura efectiva. Pero

${ }^{34}$ Ver: Memoria de .Ministerio de Interior presentada al Congreso Nacional en el año 1865, Carta de Guillermo Rawson a Manuel Lagraña, Buenos Aires, 23 de octubre de 1864; ley 531 del 18 de septiembre de 1872 de Administración y Dirección General de Ferrocarriles; Proyecto de ley sobre puentes y caminos nacionales, CNDSCD, sesión del 13 de octubre de 1875, pp. 42-45. 
además estimamos que los legisladores forjaron alianzas previas que les permitieron sumar votos y conseguir la mayoría suficiente para aprobarlas. Avanzar sobre cómo, a través de qué ámbitos y con qué objetivos se formaron esos vínculos y alianzas nos permitirá profundizar nuestro conocimiento acerca de las prácticas políticas y los múltiples canales de diálogo forjados en el proceso de inserción de las provincias al nuevo escenario nacional.

\section{Bibliografía}

Balán, Jorge. 1978. "Una cuestión regional en la Argentina: Burguesías provinciales y el mercado nacional en el desarrollo agroexportador". En: Desarrollo Económico. Revista de Ciencias Sociales, № 69, Vol. 18, Buenos Aires, Instituto de Desarrollo Económico y Social, pp. 49-88.

Barres, Francisco. 1945. Reseña de los ferrocarriles argentinos, 1857-1944. Buenos Aires: Boletín de la Asociación Internacional Permanente del Congreso Panamericano de Ferrocarriles.

Bragoni, Beatriz y Míguez, Eduardo (coord.). 2010. Un nuevo orden político. Provincias y Estado Nacional, 1852-1880. Buenos Aires, Biblos.

Bressan, Raquel. 2012. "La difícil materialización de las vías de comunicación en el litoral durante la formación del Estado-Nación (1860-1880)". En: Actas digitales del XXXII Encuentro de Geohistoria Regional. Instituto de Investigaciones Geohistóricas/ Conicet/UNNE, Resistencia, pp. 625-638.

Buchbinder, Pablo. 2004. Caudillos de pluma y hombres de acción. Buenos Aires, PrometeoUNGS.

Duarte, María Amalia. 1965. "Los ferrocarriles de Entre Ríos durante la presidencia de Sarmiento". En: Trabajos y Comunicaciones, $\mathrm{N}^{\circ}$ 13, La Plata, Universidad Nacional de La Plata, pp. 49-74.

Gómez, Teresita. 2007. "Promotores y técnicos en el tendido del Ferrocarril del Oeste". En: Mario López y Jorge Waddel (comp.) Nueva historia del ferrocarril en la Argentina, Argentina, Lumiere, pp. 295-340.

Gómez, Teresita y Schvarzer, Jorge. 2007. "Ferrocarriles, expansión agraria y distribución de la tierra. Los debates de 1860". En: Teresita Gómez, Andrés Regalsky y Jorge Schvarzer (comp.) Estudios sobre la historia de los ferrocarriles argentinos (18571940) Buenos Aires, Universidad de Buenos Aires, pp. 11-48.

Gorostegui de Torres, Haydée. 2000. La organización nacional. Buenos Aires, Paidós.

Lanteri, Ana Laura. 2011. De lo ideal a lo posible. Dirigencia e instituciones nacionales en la "Confederación" (1852-1862). Tesis Doctoral. Tandil, IEHS-UNCPBA.

López, Mario Justo. 1994. Historia de los ferrocarriles nacionales, 1866-1886. Buenos Aires, Lumiere.

Mantilla, Florencio. 2007. Bibliografia periodística de la provincia de Corrientes (1887). Corrientes, Amerindia.

Oszlak, Oscar. 1997. La formación del estado argentino. Buenos Aires, Planeta. 
Palermo, Silvana. 2006a. "Del Parlamento al Ministerio de Obras Públicas: la construcción de los ferrocarriles del Estado en Argentina, 1862-1916". En: Desarrollo Económico, No 182, Vol. 46, Buenos Aires, Instituto de Desarrollo Económico y Social, pp. $215-$ 244.

Palermo, Silvana. 2006b. "Elite técnica y Estado liberal: la creación de una administración moderna en los Ferrocarriles del Estado (1870-1910)". En: Estudios Sociales, No 30, Santa Fe, Universidad Nacional del Litoral, pp. 9-42.

Palma, Federico. 1941. Juan Eusebio Torrent. Apuntes biográficos. Corrientes.

Pérez, María Emilia. 1984. "La red vial y las comunicaciones terrestres en Corrientes. Origen y Evolución. 1588-1898”. En: Cuadernos de Geohistoria Regional, № 10, Resistencia, Instituto de Investigaciones Geohistóricas-Conicet-Fundanord.

Poenitz, Alfredo. 1987. "Proceso de ocupación espacial y poblamiento al sur del río Miriñay (1769-1869)". En: Cuadernos de Geohistoria Regional, № 18, Resistencia, Instituto de Investigaciones Geohistóricas-Conicet-Fundanord.

Poenitz, Edgard. 1981. "La ruta oriental de la yerba. Navegación y comercio en el alto río Uruguay". En: Cuadernos de Estudios Regionales, No 1, Concordia, pp. 25-60.

Poggi, Gianfranco. 1997. El desarrollo del Estado moderno. Buenos Aires, Universidad Nacional de Quilmes

Rebuelto, Emilio. 1994. Los ferrocarriles en la Argentina, 1857-1910. Buenos Aires, Fundación Museo Ferroviario.

Scalabrini Ortiz, Raúl. 1957. Historia de los ferrocarriles argentinos. Buenos Aires, Devenir.

Schaller, Enrique. 1995. "La distribución de la tierra y el poblamiento de la provincia de Corrientes (1821-1860)". En: Cuadernos de Geohistoria Regional, N³1, Resistencia, Instituto de Investigaciones Geohistóricas-Conicet-Fundanord.

Schaller, Enrique. 2002. "Las finanzas públicas de Corrientes durante la organización constitucional, 1851-1861". En: Nordeste Segunda Época, No 16, Resistencia, Universidad Nacional del Nordeste, pp. 5-29.

Schmit, Roberto. 1999. "Fronteras rioplatenses: Ocupación del espacio y estructura socioocupacional en el oriente entrerriano (1820-1850)". En: Jorge Gelman, Juan Carlos Garavaglia y Blanca Zeberio (comp.) Expansión capitalista y transformaciones regionales, Buenos Aires, La Colmena/ Instituto de Estudios Histórico-Sociales, pp. 45-74.

Schmit, Roberto. 2003. "Los caminos al mercado en el Alto Uruguay: comercio y política mercantil del oriente entrerriano en tiempos de guerra". En: Anuario del Centro de Estudios Históricos" Prof. Carlos S. A. Segreti, № 2-3, Córdoba, Instituto de Estudios Históricos" Prof. Carlos S. A. Segreti, pp. 101-124.

Schmit, Roberto. 2007. Los límites del progreso: expansión rural en los orígenes del capitalismo rioplatense. Buenos Aires, Siglo XXI.

Vanossi, Roberto. 2007. "Dos exponentes del ideario liberal en la Generación del 80”. En: Academia Nacional de Ciencias Morales y Políticas, Buenos Aires. 
Bressan. El Congreso Nacional y los proyectos para la construcción...

\section{ANEXO 1}

Rutas de comercialización en la segunda mitad del siglo XIX

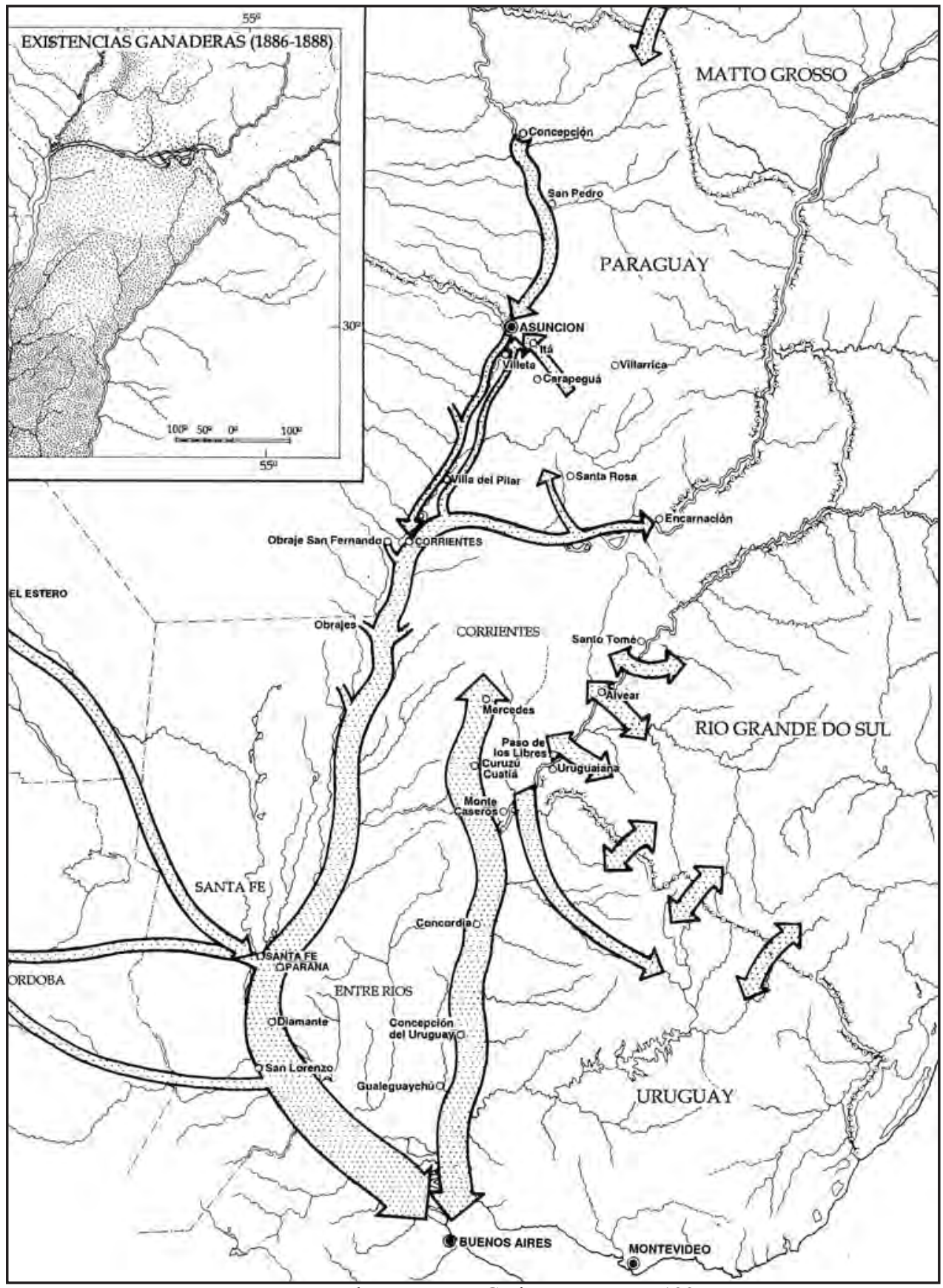

Fuente: Maeder, Ernesto y Gutierrez, Ramón. 1995.

Atlas Histórico del Nordeste Argentino.

Resistencia, Instituto de Investigaciones Geohistóricas/Conicet/Fundanord 